\title{
The effect of absorption process parameters on its efficiency
}

\author{
Valeri Kiss ${ }^{1, *}$, Aleksandr Evdokimov ${ }^{1}$, Tatiana Kaverzneva $^{2}$, and Dmitriy Tarkhov ${ }^{2}$ \\ ${ }^{1}$ Saint Petersburg National Research University of Information Technologies, Mechanics and Optics, \\ Kronwerkski 49, St. Petersburg, 197101, Russian Federation \\ ${ }^{2}$ Peter the Great St. Petersburg Polytechnic University, Polytechnicheskaya 29, St. Petersburg, \\ 195251, Russian Federation
}

\begin{abstract}
In this work we are presenting the results of studies of the effect of hydro and aerodynamic parameters of the process of water vapor absorption during intensive bubbling in the dynamic foam mode on the absorption process efficiency. As a result, it was found that the hydrodynamic characteristics (height of the foam layer of the absorbent and its resistance) and aerodynamic characteristics (gas velocity, longitudinal mixing in the gas phase) have the main influence on the efficiency of the absorption process. We also investigated the effect on the efficiency of the process of kinetic characteristics, characterized by the number of transport units and absorption factor. We have proposed to estimate the overall efficiency of the process, taking into account the degree of extraction, as well as the energy characteristics (hydraulic resistance), weight and size characteristics and the drop entrainment volume. For this purpose, for the overall assessment we have proposed to introduce a conditional optimality ratio, allowing to make a comparison of both various contact devices and absorbents.
\end{abstract}

\section{Introduction}

The systems of both comfortable and technological air conditioning, as a rule, include dehumidification units $[1,5]$. Most widely used are the air dryers based on the use of various sorbents [6]. The choice of the sorbent depends primarily on the requirements for the degree of dehumidification. In addition, it is necessary to take into account the energy efficiency of the process, the weight and size characteristics of the drop entrainment and a number of other factors.

This work is devoted to the study of the process of air dehumidification using liquid sorbents. The efficiency of absorption processes depends to a large extent on the catalyst chamber in which this process is carried out.

\footnotetext{
*Corresponding author: vvkiss@yandex.ru
} 


\section{Methods}

For the catalyst chamber we have chosen a cyclone-foam apparatus with the dynamic foam mode. Devices of this type have proved their high efficiency when conducting heat and mass transfer processes [7-9].

In order to identify the optimal operating modes of the cyclone-foam apparatus, a study was conducted of the effect of various process parameters on the efficiency of absorption.

In the process of the study, the following basic parameters were measured: air velocity and flow rate, consumption of absorbents, air and absorbents temperature, moisture content of air, concentration of absorbents, height of the dynamic foam layer.

The efficiency of the absorption apparatus is usually characterized by the degree of the component extraction [10].

The degree of extraction achieved depends on the technological mode of operation of the apparatus and on its quality [11-16]. The degree of extraction can be expressed by the extraction ratio, which is the ratio of the amount of the component actually absorbed to the amount that would be absorbed with the most complete extraction:

$$
\psi=\frac{C_{0}^{\Gamma}-C_{1}^{\Gamma}}{C_{0}^{\Gamma}-C_{1}^{\Gamma^{*}}},
$$

where $C_{0}^{T}$ is the initial concentration of the component in the incoming gas, $\mathrm{kg} / \mathrm{m}^{3} ; C_{1}^{\Gamma}$ is the final concentration of the component in the incoming gas, $\mathrm{kg} / \mathrm{m}^{3} ; C_{0}^{\Gamma^{*}}$ is the concentration of the component in the gas, in equilibrium with the concentration of the incoming liquid, $\mathrm{kg} / \mathrm{m}^{3}$.

A number of authors use the ratio of the overall efficiency of mass transfer according to Murphree [17] as a characteristic of the efficiency of mass transfer:

$$
E=\frac{C_{0}^{\Gamma}-C_{1}^{\Gamma}}{C_{0}^{\Gamma}-C_{0}^{\Gamma^{*}}}
$$

where $C_{0}^{\Gamma}$ is the concentration of the component in the gas, in equilibrium with the concentration of the outgoing liquid, $\mathrm{kg} / \mathrm{m}^{3}$.

From equations (1) and (2) it follows that the value of the extraction ratio $\psi$ determines the composition of the outgoing gas at the initial concentration of the component in the incoming gas $C_{0}^{\Gamma}$ and the incoming liquid $C_{1}^{\cdots}$ and the value of the total mass transfer efficiency $E$ determines the composition of the outgoing gas at the initial concentration of the component in the incoming gas $C_{0}^{\Gamma}$ and outgoing fluid $C_{0}^{\varkappa c}$.

Equations (1) and (2) are related by the formula:

$$
E=\frac{\psi}{1-\frac{\psi}{A}}
$$

where $A=\frac{V_{\varkappa c}}{V_{\Gamma} m_{c}}$ is the absorption factor; $V_{\varkappa}$ - liquid flow rate, $\mathrm{m}^{3} / \mathrm{h} ; V_{\Gamma}$ - air flow rate, $\mathrm{m}^{3} / \mathrm{h} ; m_{c}$ - is the equilibrium constant. 
Evaluation of the efficiency of mass transfer using the ratio $E$ is convenient for directflow phase motion, since in this case, $E$ it determines the efficiency of the process as a measure of its approximation to the theoretical one.

\section{Results and Discussion}

As a characteristic of the efficiency of the absorption process in a cyclone-foam apparatus, it seems appropriate to use an extraction factor of $\psi$, which depends on the number of transfer units $N_{o 2}$.

In order to achieve the most efficient operation of the apparatus for a given number of transfer units $N_{o z}$, the absorption factor value must be greater than 60 .

The degree of extraction also depends on the kinetics of the process, expressed by the number of transfer units $N_{o z}$ and on the degree of longitudinal mixing, characterized by the Peclet number. The influence of these parameters is unequal for different values of the absorption factor; therefore, it seems appropriate to consider the dependences for little ( $A$ $=\mathrm{I})$ and $\operatorname{big}(A=60)$ values of the absorption factor, as is usually done in similar studies.

With the absorption of water vapor in conditions of low values of the absorption factor $A$, longitudinal mixing produces almost no effect on the value of the extraction ratio. The extraction ratio increases with an increase in the number of transfer units up to $N_{o 2}$. $=2.0$. A further increase in the contact intensity has very little effect on the value of the extraction ratio.

The extraction ratio in this case characterizes to a greater extent the quantitative side of the process or the depth of drainage.

With perfect mixing of the liquid that takes place in the cyclone-foam apparatus, the qualitative side of the process of mass transfer, i.e. the degree of its approximation to the theoretically possible, most fully reflects the ratio of the overall mass transfer efficiency according to Murphree $E$. The values of the ratio of overall mass transfer efficiency according to Murphree $E$ and the extraction ratio $\psi$ are strongly influenced by longitudinal mixing in the dispersed phase, which substantiates the need to be taken into account when calculating the processes of absorption of water vapor during intensive bubbling carried out in a cyclone-foam apparatus.

The kinetics of the absorption process strongly depends on the mode of operation of the apparatus. To establish the optimal mode of the absorption process, it seems reasonable to consider the direct influence of the regime parameters on the value of the ratio $E$.

The dependence of the overall efficiency of the absorption process on the air velocity has a maximum. The calculation established that the maximum value of the ratio of the overall efficiency of the mass transfer process according to Murphree corresponds to such a mode of operation of the cyclone-foam apparatus, in which the gas content of the dynamic foam layer is 0.74 .

With a further increase in air velocity in the apparatus, the absorption efficiency first decreases and then at a speed of more than $4.5 \mathrm{~m} / \mathrm{s}$ it becomes almost constant. At low altitudes of the gas-liquid layer, even a slight increase in $E$ is observed. The absence of the influence of the gas velocity on the intense regimes indicates that the internal circulation in gas bubbles reaches such values that it becomes able to compensate for the decrease in the specific contact surface of the phases; while the efficiency of the process is determined mainly by the contact time, i.e. height of the dynamic foam layer. With increasing height of the dynamic foam layer, the overall absorption efficiency increases. 
The effect of foam height is most pronounced at $H_{n}<0.3 \mathrm{~m}$. With a further increase in the height of the gas-liquid layer, its effect on the absorption efficiency weakens. The optimal height of the foam in a cyclone-foam apparatus can be considered as $H_{n}$ $0.3+0.35 \mathrm{~m}$.

The maximum possible efficiency of the mass transfer process, depending on the height of the dynamic foam layer, is achieved at gas velocities in the working zone of the cyclonefoam apparatus equal to $1.5-2.7 \mathrm{~m} / \mathrm{s}$. However, with increasing gas velocity, up to e.g. 5 $\mathrm{m} / \mathrm{s}$, the total process efficiency decreases by $10+12 \%$. The dimensions of the device are reduced by about 2 times. At the same time, as is known [18, 19], when the gas velocity changes, the hydraulic resistance of the apparatus also changes and, as a result, the energy indices change. The velocity of the gas and the height of the dynamic foam layer also affect the size of the drop entrainment volume [20,21].

Accounting for such a variety of factors affecting the overall efficiency of the apparatus is advisable to be produced using the conditional optimality ratio [22], which can be presented in the following form:

$$
k_{\text {onm }}=\frac{\Delta C}{S H_{n} e \Delta P},
$$

where $\Delta C$ is the amount of absorbed water vapor, $\mathrm{kg} / \mathrm{m}^{3} ; \Delta P$ is the resistance of the apparatus, $\mathrm{Pa} ; e$ is the amount of the blown solution, $\mathrm{kg} / \mathrm{m}^{3} ; S$ is the area of the apparatus, $\mathrm{m}^{2} ; H_{n}$ is the height of the foam layer, $\mathrm{m}$.

Figure 1 shows the calculated dependence of the optimality criterion on the gas velocity and the height of the dynamic foam layer for the case of absorption of water vapor from the air with a solution of lithium bromide.

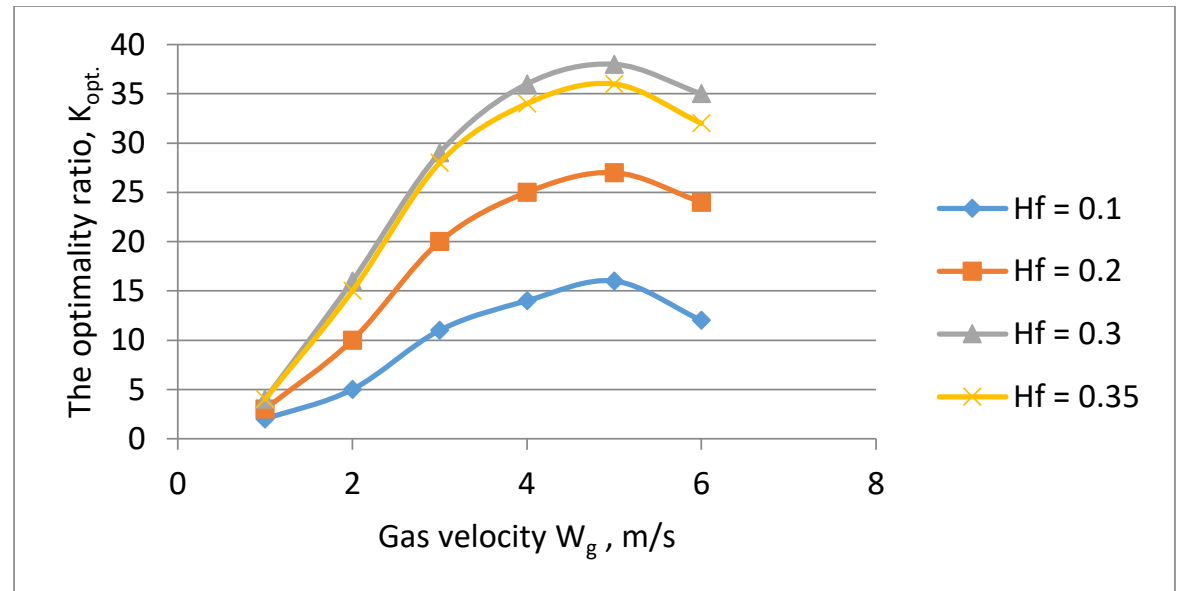

Fig. 1. The dependence of the value of the optimality ratio on the gas velocity and the height of the layer of dynamic foam for the $\mathrm{LiBr} /$ solution

The dependencies for the other solutions studied are of a similar nature. As can be seen from Fig. 1, the optimal value of the gas velocity in a cyclone-foam apparatus is $5 \mathrm{~m} / \mathrm{s}$. In addition, growth of the optimality criterion is observed to the dynamic foam layer height values $H_{n}-0,3 \mathrm{~m}$.

The ratio of optimality can also be used for a comparative evaluation of the efficiency of the use of various water-absorbing solutions. 


\section{Conclusions}

The results of the study showed that the maximum value of the optimality ratio for all four absorbents corresponds to a gas velocity of $5 \mathrm{~m} / \mathrm{s}$ and a height of the dynamic foam layer of $0.3 \mathrm{~m}$.

A solution of lithium chloride is the most effective for the air dehydration process, followed by choline chloride, lithium bromide and calcium chloride. Efficiency of lithium bromide is in third place, although it has the highest absorption capacity. This is primarily due to its high density, which, accordingly, strongly affects the resistance of the gas-liquid layer and the overall efficiency of the process.

By optimality ratio choline chloride solution is only $16 \%$ inferior the lithium chloride solution, however, it is almost nine times cheaper than lithium chloride salts and has virtually no corrosive effect on aluminum-magnesium alloys.

Thus, using the optimality ratio, it is possible to estimate the overall efficiency of the absorption process, taking into account the extraction ratio, as well as such important characteristics as flow resistance affecting energy consumption, dimensions and, correspondingly, metal consumption of the apparatus and drop entrainment. Also, with the help of the optimality ratio, it is possible to compare various absorbents by their overall efficiency.

\section{References}

1. A. V. Tsygankov, A. M. Grimitlin, Vestnik Mezhdunarodnoi akademii kholoda, 4, 4750 (2013).

2. S.A. Sergunin, S. I. Simanenkov, N. T. Gatapova, Bulletin of Tambov state technical University, 1, 197-202 (2012).

3. D. Baranova, D. Sovetnikov, D. Semashkina, and A. Borodinecs, Procedia Eng. 205, 503 (2017).

4. A. Nefedova, D. Chernyshev, and D. Tseytin, Procedia Eng. 117, 1112 (2015).

5. A. Suslova, M. Petrichenko, and A. Sivokhin, MATEC Web Conf. 73, 2008 (2016).

6. N. B. Kochenkov, Vestnik Mezhdunarodnoi akademii kholoda, 1, 51-56 (2013).

7. S. A. Bogatykh, Cyclone-foam machines, Mashinostroenie, (1978).

8. V.V. Kiss, I. E. Radionova, National Association of scientists, 4, 33-36 (2014).

9. V. V. Kiss, Processes and Food Production Equipment, 2 (2014).

10. V. M. Ramm, Adsorption of gases. Moscow, Chemistry, (1976).

11. V. P. Zhukov, Vesti IGEU, 4, 12-16 (2012).

12. L. E. Osipova, E. Sh. Teplyakov, D. A. Amineva, B. N. Matyushko, Vestnik Kazanskogo tekhnologicheskogo universiteta, 22, 9-14 (2012).

13. S. Yu. Andreev, G. P. Davydov, A. A. Petrunin, Proceedings of The international Symposium «Reliability and quality», 1, 194-196 (2012).

14. V. E. Nakoryakov, N. I. Grigoryeva, M. V. Bartashevich, Int. J. Heat and Mass Transfer, 21, 4485-4490 (2011).

15. B.V. Dzybenko, L-V. Ashmantes, M.D. Segal, New York: Begell House Jnc. p. 200 (2000).

16. V.A. Kaminskii, N.Y. Obvintseva, Rus. J. Phys.Chem., 7, 1215-1220 (2008).

17. Yu. D. Maksimenko, Bulletin of Astrakhan state technical University, 1 (20) (2004). 
18. A. A. Sidyagin, A. V. Stepikin, I. A. Balakhnin, Modern problems of science and education, 6, 176-183 (2014).

19. A. F. Sorokopud, D. S. Strizhkov, V. S. Kozymaev, Equipment and technology of food production, 4, (27) (2012).

20. A. T. Ismagilov, Oil and Gas business, 1, 32 (2010).

21. V.I. Alfyorov, L.A. Bagirov, L.M. Dmitriev, V. Feygin, S. Imayev, Oil \& Cas Journal, 23, 53-58 (2005).

22.V.V. Kiss. Proceedings of VII international sciencetechnical conference "Lowtemperature and food technologies in XXI century». SPb.: ITMO University, 43-45 (2015).

1 D. Baranova, D. Sovetnikov, D. Semashkina, and A. Borodinecs, Procedia Eng. 205, 503 (2017).

2 A. Nefedova, D. Chernyshev, and D. Tseytin, Procedia Eng. 117, 1112 (2015).

${ }^{3}$ A. Suslova, M. Petrichenko, and A. Sivokhin, MATEC Web Conf. 73, 2008 (2016). 